\title{
VidShare: A Management Platform for Peer-to-Peer Multimedia Asset Distribution Across Heterogeneous Access Networks with Intellectual Property Management
}

\author{
Tom Pfeifer, Paul Savage, Jonathan Brazil, and Barry Downes \\ Telecommunications Software \& Systems Group [TSSG] \\ Waterford Institute of Technology [WIT] - Carriganore Campus \\ Waterford, Ireland \\ Phone: +353-51-30-2927 \\ t.pfeifer@computer.org, \\ \{psavage, jbrazil, bdownes\} atssg.org
}

\begin{abstract}
The VidShare project develops a management platform for Peer-toPeer multimedia asset distribution across heterogeneous access networks, fixed and mobile, with Intellectual Property Management and Protection (IPMP). A key objective is to build an architecture that is interoperable with different content management and IPMP solutions. The project utilises MPEG REL as a rights expression language and works with the evolving MPEG-21 Multimedia Framework. The platform supports a number of business models including rights based personal multimedia sharing, distributed multimedia management for corporations, and the retailing of video content including the super distribution of content.
\end{abstract}

Keywords: multimedia in peer-to-peer networks, mobile multimedia, mobile network management, digital rights management, multimedia middleware and frameworks, content distribution networking.

\section{Introduction}

Currently, multimedia technology provides the different players in the multimedia value and delivery chain (from content creators to end-users) with access to information and services from almost anywhere at anytime through ubiquitous terminals and networks. However, no complete solutions exist that allow different communities, each with their own models, rules, procedures, interests and content formats, to interact efficiently in complex, automated e-commerce scenarios.

In addition most multimedia content is not governed today by Intellectual Property Management and Protection (IPMP) systems. Where they do exist they are rudimentary and do not interoperate. This creates a major barrier to rolling out complex multimedia services that require this interoperability and respect for Intellectual Property Rights (IPR), such as media sharing, retailing or superdistribution of video content.

The VidShare ${ }^{1}$ platform identifies and defines the key elements needed to support these multimedia services and their delivery chain as described above, the relationships

\footnotetext{
1 The VidShare project has been supported by Enterprise Ireland within the Commercialisation Fund - Technology Development Phase 2005, TD/2005/229, 2005 - 2006.
} 
between and the operations supported by them. Thus the key objective of the VidShare project was to research and develop a peer-to-peer e-commerce platform, that enables the distribution of multi-media content (with a particular emphasis on video) across heterogeneous access / distribution networks (Internet, Mobile, Digital TV) with a management framework that enables interoperability of content and IPMP systems.

The key differentiator that VidShare has over other P2P networks is its focus on standards based multimedia interoperability and Intellectual Property (IP) management and protection. Participants in a P2P network can bring lots of legitimate value to both IP owners and users.

Within this paper, we discuss current limitations in Section 2. The platform design and architecture is introduced in Section 3, starting with a scenario based analysis, the architectural overview, and the embedding of IPMP and e-commerce functionality. Section 4 presents aspects of the implementations, and in Section 5 we summarise.

\section{Key Issues and Existing Technical Limitations}

When looking at the situation of how multimedia content is managed and protected today, the key issues and technical limitations of existing products, processes and methodologies today are:

1. Current content management systems do not communicate with each other; content cannot readily be identified across content management systems, or accessed in an easy-to-use, distributed fashion.

2. Most of the multimedia content existing today is governed by at best rudimentary IPMP systems.

3. No IPMP system has yet emerged as a de-facto standard. While various IPMP systems exist today, no management framework exists to allow for interoperation amongst such systems.

4. One problem for End Users interacting with content today is the lack of interoperability between IPMP systems.

5. Users currently need to have very explicit format and technology knowledge in order to utilise format conversion tools to use content on different devices.

6. Owners of rights in content require the freedom to exercise their rights by choosing channels and technologies (including IPMP Systems) through which to offer and make available their content.

7. There is a lack of standardised methods for monitoring and detecting infringements of rights.

8. Consumers of content may in some circumstances require the freedom to manage their privacy, which includes interacting with content without disclosing their identity to any other User in the value chain; Note that through new technologies (e.g., the Internet), End Users increasingly become owners of rights in content.

9. The differences between the national and regional legislations relating to IP law (and the lack of any universal legislation to protect rights holders) challenge existing IPMP systems to accommodate the evolution of global commerce in a digital environment.

10. The lack of integration of P2P networks with IPMP systems. 


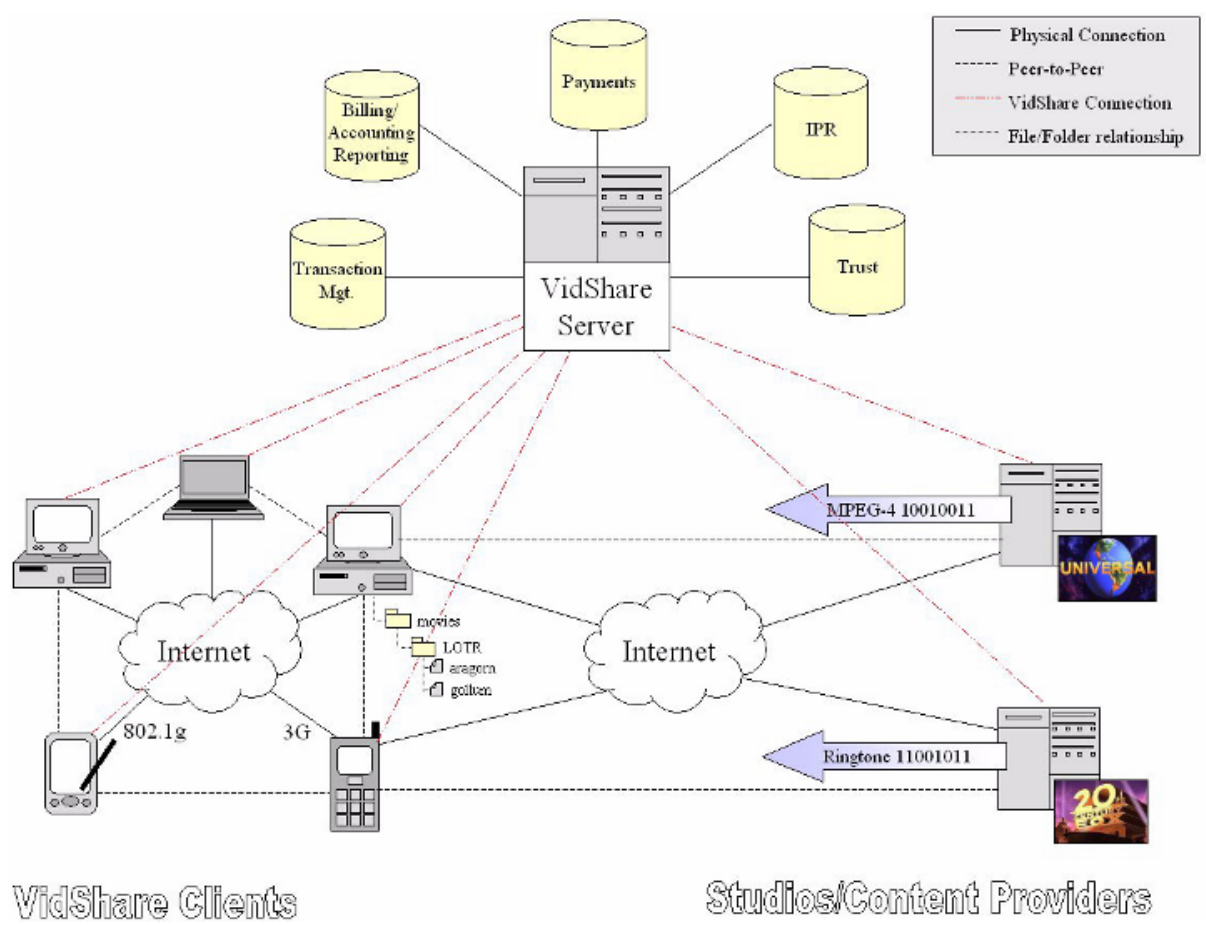

Fig. 1. VidShare high level architecture

\section{Design and Architecture}

\subsection{Scenario Based Analysis}

A key objective of the VidShare platform is to facility a number of innovative peer-topeer based digital business models in a secure protected way. The Scenario Based Approach [1] has been used to understand the operation between owners providers and users of video content. The usage scenarios have been defined as follows:

1. Rights based personal media sharing - e.g. one user sharing personal video content, such as a family occasion, with the other family members in a secure, rights based manner (i.e. named users only can play the video, no editing or forwarding - a time limit of 1 month is specified).

2. Rights based distributed corporate multimedia database - for example the Dublin office creating and locally hosting content that the New York office can securely access and consume and visa versa. With rights based access, content can also be restricted by, for example, organization security clearances.

3. Rights based e-tailing of video content. In this scenario users will have the capability of searching for and then downloading and playing movies such as "The Day After Tomorrow". In this scenario the user has the right to play a trailer for the movie and then has the option to rent or buy the movie. 
4. The super distribution of video content. In this scenario the content the user has downloaded has rights that enable the content to be forwarded to another user. A second user should be able to play the trailer and then have the option to rent or buy the movie. If the second user makes a purchasing decision the platform should have the flexibility to enable the original user to receive a percentage of the purchase price as a reward, in addition to settling with the players in the distribution chain.

\subsection{Architecture}

To enable the business models discussed in the scenarios above, the VidShare project implements the following technological components, as illustrated in Figure 1.

A distributed Peer-to-Peer architecture (P2P). This is a pure peer-to-peer network similar to the architecture of Gnutella for person-to-person and organisationperson sharing of secure rights based multimedia. This network requires augmentation with the VidShare management system to support the retailing and superdistribution of value based content to enable, content identification and IPR verification, the establishment of Trust relationships, value chain support, payments, accounting and settlement.

A client P2P application that supports:

1. User profile and preferences, content searching and downloading.

2. Functionality to enable a user to upload their personal video and encode/decode it with rights using the MPEG-21 REL standard.

3. Functionality to create a local, standards based, media database of sharable content for Peer-to-Peer sharing.

A management platform that oversees the Peer-to-Peer network which supports:

1. A framework to manage the $\mathrm{P} 2 \mathrm{P}$ network including a register of peers.

2. A central index database for organisations and/or media companies where IPR validated content can be listed. Note: for legal reasons, private individual's content databases will be held locally and not in the central index database.

3. Value Chain Management and License services. This includes content License generation and distribution, content identification (and verification of IPR), content tracking and business model services such as superdistribution.

4. Trust Management Services. Registration of value chain participants, user certification and credential management services, trusted time services; in connection with Security and Protection Services. Secure channels, key management, trusted software and execution environment.

5. An e-commerce framework: Payments services, accounting services and settlement services; together with transaction, audit and log services. These services can be monitored by content providers and rights holders for auditing purposes.

\subsection{IPMP Integration}

VidShare utilises MPEG REL as a key element of its IPMP architecture, as MPEG is working with other organisations such as the OMA to integrate their approaches and ensure interoperability. Using the MPEG REL for content distribution provides the following benefits: 
- It allows content distribution terms and conditions in precise XML-based language for both human and machine interpretation.

- It empowers content owners to manage the rights and conditions throughout the distribution channels, down to the level of content consumers, through the specification of the multi-tier distribution relationships, potentially in a single multi-tier MPEG REL license.

- It facilitates e-commerce by enabling the upstream content provider to present multiple offers (choices) to the downstream distributors or consumers, with the final selection presented as an enforceable license.

- It helps to automate the content distribution process by matching up the supply and demand entities based on contractual permissions and obligations.

- It enables the specification of any type of licenses, grants, rights, and conditions, by providing a comprehensive and extensible language. It provides unambiguous interpretation and interoperable expressions of MPEG REL licenses.

- It enables interoperability, and positions players in the content distribution value chain to use of best of breed components or web services and support interoperability across business models.

MPEG REL can be integrated directly into MPEG-4's binary format through MPEG4's IPMP Hooks. The underlying philosophy is that the bitstream embeds information that informs the application which (of possibly multiple) IPMP system should be used to process governed objects in compliance with rules declared by the metadata. MPEG-4 integrates the hooks tightly with the MPEG-4 Systems layer, which makes it possible to build secure MPEG-4 delivery chains very efficiently.

There are two key extensions of basic MPEG-4 systems constructs:

- IPMP-Descriptors (IPMP-Ds) are part of the MPEG-4 object descriptors that describe how an object can be accessed and decoded. They are used to denote the IPMP System that was used to encrypt the object.

- IPMP-Elementary Streams (IPMP-ES) are all MPEG objects represented by elementary streams, which can reference each other. These special elementary streams can be used to convey IPMP specific data.

Using these tools, it is possible to have the IPMP architecture act on the media data; the scene description and the compositor; the interaction messages; and any other information conveyed in the BIFS (Binary Format for Scenes, MPEG-4's binary scene description / animation language).

\subsection{E-Commerce and Management Capabilities}

In addition to the IPMP architecture, VidShare integrates e-commerce capabilities into the management platform to support the project's business models. The key elements being a payments system, accounting system and settlement system to enable consumers to purchase and pay for content (and for content providers to receive the appropriate out-payments on sales of content).

As the key elements of this project relate to the P2P distribution of content with IPMP, exiting technology is integrated for the e-commerce framework rather than built for this project. 
Thus the project integrates PayPal (www.paypal.com) for payments (which can work in a P2P environment) and the TSSG's Rating Bureau Service (RBS) for accounting and settlement.

\section{Implementation}

\subsection{Components}

The implementation of the VidShare system has been modularised so that custom configurations are possible for each target customer. Following is a detailed description of each module within the VidShare platform.

\subsection{Mediation Component}

The mediation component of the VidShare platform is responsible for the generation and hand-off of service usage records. These service usage records will detail each transaction authorised by the VidShare platform. A sample usage document is provided in Figure 2, adopting an XML format to keep the mark-up in a transportable and easily readable format.

The current mode of operation for the mediation component is to operate as the 'D' interface of the Network Data Management-Usage (NDM-U) specification [10]. This interface has been adopted as the immediate testing harness for the accounting aspect of the platform utilises the Rating Bureau Service (RBS), a TSSG rating service that exposes NDM-U interfaces and is IPDR compliant.

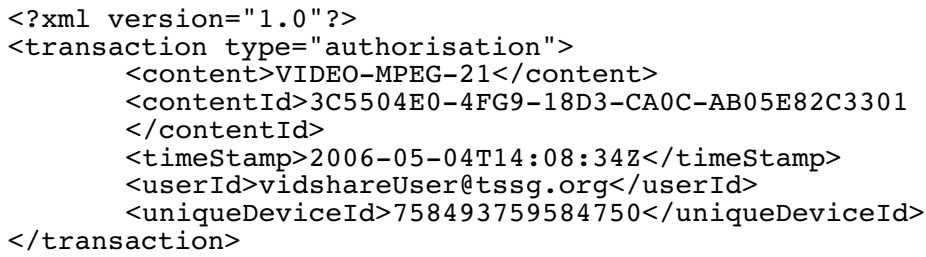

Fig. 2. Sample instance document for a VidShare transaction

\subsection{IPMP Component}

The Intellectual Property Management and Protection (IPMP) component is responsible for interpreting the requests issued for authorisation of content by client devices. It is responsible for generating the correct licence, associated with the content being authorised. Initially this IPMP component has been developed to support the MPEG-21 Rights Expression Language (REL) [5]. However, it is viewed that this component will be enhanced to be compatible with the rights expression language of the Open Mobile Alliance (OMA). 


\subsection{Transaction Management}

The transaction management component is currently a baseline implementation of transaction management functionality using the transaction management features of J2EE. As VidShare moves toward a commercial deployment it is viewed that this component will be replaced by a more substantial transaction manager (e.g. Tuxedo) as used by operators for their current customer services.

\subsection{Trust Management}

Trust management is critical to the successful operation of the VidShare platform. It is imperative that customers are assured of the security of their transactions; that content which they are acquiring is originating for a legitimate source and that any content or licence files distributed to them is verifiably safe and traceable. Without these measures in place, users of the system will not feel comfortable using content and licence providers that may be distributing malware or illegal content.

\subsection{Payments Component}

In order to fulfil the overall end-to-end provisioning of the service a basic payments solution has been implemented which uses the PayPal API to engage a micropayment solution for content e-tailing. PayPal serves as a useful mechanism for ensuring the security of users transactions and user experience by invoking a trusted third party payments processor. Again, it is envisaged that as VidShare moves toward a commercial deployment, this payment processor will be replaced by the in-house engines used by the mobile operators or other target customers.

\subsection{VidShare Positioning}

The VidShare platform is being positioned in the market as a plug-in solution for operators' networks. It will provide a standards compliant, black box interface for solving the DRM needs of network operators and the content providers who offer services over them. It is not viewed that VidShare will become a standalone service provisioning platform such as those currently seen in the Web 2.0 service domain (e.g. flickr.com) but rather it will be the behind the scenes enabler for mobile content distribution and super-distribution.

\section{Summary and Future Work}

VidShare is a Peer-to-Peer, e-commerce platform and client. It will enable content providers, such as 20th Century Fox, Universal, MTV, to distribute their video content to consumers in a secure environment to different types of devices such as PC's, mobile phones and evolving hybrid devices such as wireless multimedia terminals.

A key aspect of VidShare is that it offers the maximum distribution flexibility that a P2P network offers - but at the same time is controlled by a Intellectual Property Management and Protection (IPMP) system the supports the secure delivery of 
protected content, i.e. it prevents the content from being stolen or counterfeited. VidShare's management system also handles payment, transaction management, auditing, reporting, royalty and revenue settlement. VidShare in essence can automate a whole series of multimedia e-commerce business models.

Security issues are investigated in close cooperation with European Commission funded 6th Framework projects coordinated by the TSSG: SEINIT and SecurIST. We plan to fully exploit the results of the VidShare research by establishing a Campus Company which will revise the prototype as a commercial grade $\mathrm{P} 2 \mathrm{P}$ system for marketing and distribution to the global marketplace.

\section{References}

[1] Kentaro Go; John M. Carroll: The blind men and the elephant: views of scenario-based system design. - interactions. ACM Press, New York, Vol. 11 (6) Nov+ Dec 2004, pp. 44-53

[2] ISO/IEC TR 21000-1, Vision, Technology and Strategy, July 2001 (DTR, N4333) and ISO/IEC PDTR 21000-1 Second Edition, Vision, Technology and Strategy, Dec 2003 (N6269).

[3] ISO/IEC IS 21000-3, Digital Item Identification, July 2002 (N4939).

[4] MPEG-21 Requirements Document V.2, December 2003 (N6264).

[5] MPEG-21 Rights Expression Language: Enabling Interoperable Digital Rights Management, Xin Wang, John R. Smith, IEEE Multimedia, October-December 2004, Vol 11, No. 4

[6] ISO/IEC FDIS 21000-7, Digital Item Adaptation, December 2003 (N6168).

[7] U.S. Department Of Defense. Technical Architecture Framework For Information Management (TAFIM) Volumes 1-8, Version 2.0. Reston, VA: DISA Center for Architecture, 1994. http://www-library.itsi.disa.mil/tafim/tafim.html (1996).

[8] ISO/IEC 14496-1 FDAM-3, IPMP extensions on MPEG-4 systems, October 2002 (N5282)

[9] ISO/IEC FDIS 13818-11, IPMP extensions on MPEG-2 systems, March 2003 (N5608)

[10] IPDR Business Solution Requirements - Network Data Management-Usage (NDM-U) v3.5.0.1, IPDR.org, November 2004 